\title{
Malignant Peripheral Nerve Sheath Tumors of the Pericardium in a Patient with Neurofibromatosis Type 1: The Diagnostic Value of ${ }^{18}$ F-FDG PET/CT and ${ }^{123}$ I-MIBG SPECT/CT
}

\author{
Mathieu Charest ${ }^{1}$, Josephine Pressacco ${ }^{2}$, and Jaramie Thomas-Gittens ${ }^{3}$ \\ ${ }^{I}$ Nuclear Medicine Department, Hôpital du Sacré-Coeur de Montréal, Montréal, Canada; ${ }^{2}$ Radiology Department, Hôpital du \\ Sacré-Coeur de Montréal, Montréal, Canada; and ${ }^{3}$ Radiology Department, Université de Montréal, Montréal, Canada
}

\begin{abstract}
A 25-y-old woman with known neurofibromatosis type 1 and a large anterior mediastinal mass was investigated. ${ }^{18} \mathrm{~F}-\mathrm{FDG}$ $\mathrm{PET} / \mathrm{CT}$ revealed a radiotracer-avid anterior mediastinal mass having an SUV $\mathrm{Vax}_{\max }$ of 4.3 and demonstrating a hypoactive center. The ${ }^{123} \mathrm{I}$-metaiodobenzylguanidine SPECT/CT study performed subsequently did not demonstrate any uptake, thereby excluding, for the most part, the diagnosis of paraganglioma or neuroblastoma. At final pathology, a malignant peripheral nerve sheath tumor of the pericardium with areas of chondrosarcomatous and angiosarcomatous differentiation was diagnosed.
\end{abstract}

Key Words: malignant peripheral nerve sheath tumors; neurofibromatosis type 1; pericardium; anterior mediastinum; FDG; PET/CT

J Nucl Med Technol 2018; 46:61-62

DOI: 10.2967/jnmt.117.199927

\section{$\mathbf{W}$}

e report the case of a woman with known neurofibromatosis type 1 and a large anterior mediastinal mass in whom the combination of ${ }^{18} \mathrm{~F}-\mathrm{FDG}$ and ${ }^{123}$ I-metaiodobenzylguanidine allowed us to correctly orient the diagnosis toward a primary malignant tumor of the pericardium and characterize its heterogeneity.

\section{CASE REPORT}

A 25-y-old woman with known neurofibromatosis type 1 came to the emergency room complaining of palpitations and chest pain. The initial work-up, including thoracic CT, revealed a large pericardial collection. After pericardiocentesis, a large anterior mediastinal mass was demonstrated by cardiac ultrasound, leading to further investigation.

${ }^{18}$ F-FDG PET/CT images (Figs. 1A and 1B), along with corresponding slices of a contrast-enhanced thoracic CT (Fig. 1C), revealed a radiotracer-avid anterior mediastinal

Received Jul. 26, 2017; revision accepted Sep. 11, 2017.

For correspondence or reprints contact: Mathieu Charest, Hôpital du Sacré-Coeur de Montréal, 5400 boul. GouinOuest, Montréal, Québec, Canada, H4J 1 C5.

E-mail: mathieu.charest@gmail.com

Published online Nov. 10, 2017.

COPYRIGHT (C) 2018 by the Society of Nuclear Medicine and Molecular Imaging. mass having an $\mathrm{SUV}_{\max }$ of 4.3 and demonstrating a hypoactive center. The tumor-to-liver ratio was measured at 2.85. The mediastinal mass was deemed malignant on the basis of these findings. No distant active lesion was detected.

The ${ }^{123}$ I-metaiodobenzylguanidine SPECT/CT study (Figs. 1D and 1E) performed subsequently did not demonstrate any radiotracer uptake by the mediastinal mass, thereby excluding, for the most part, the diagnosis of paraganglioma or neuroblastoma.

At final pathologic examination of the surgically removed lesion, a malignant peripheral nerve sheath tumor of the pericardium with areas of chondrosarcomatous and angiosarcomatous differentiation was diagnosed.

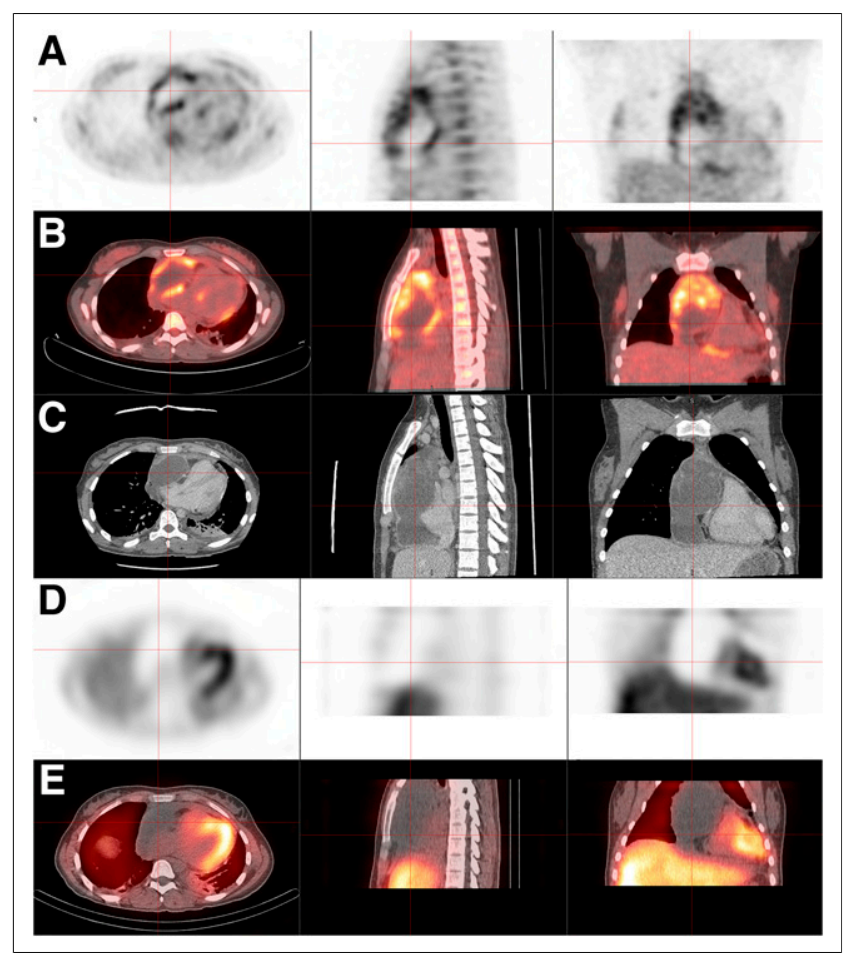

FIGURE 1. ${ }^{18} \mathrm{~F}-\mathrm{FDG}$ PET (A), PET/CT (B), and CT (C) reveal radiotracer-avid anterior mediastinal mass with hypoactive center. ${ }^{123}$-metaiodobenzylguanidine SPECT (D) and SPECT/ CT (E) show no radiotracer uptake by mediastinal mass. 


\section{DISCUSSION}

Neurofibromatosis patients carry up to a $10 \%$ lifetime risk of developing a malignant peripheral nerve sheath tumor, although this type of tumor is rare in the general population $(<0.1 \%)(1) .{ }^{18} \mathrm{~F}-\mathrm{FDG}$ PET/CT was found to be highly sensitive and specific for the diagnosis of malignant peripheral nerve sheath tumor in neurofibromatosis type 1 patients (1-3). Warbey et al. reported a sensitivity of 0.97 and specificity of 0.87 using a cutoff $\mathrm{SUV}_{\max }$ of 3.5 (4). Salamon et al. proposed a tumor-to-liver ratio of more than 2.6 as being more specific (5). Both criteria were met in our case.

\section{CONCLUSION}

The combination of ${ }^{18} \mathrm{~F}$-FDG and ${ }^{123}$ I-metaiodobenzylguanidine allowed us to correctly orient the diagnosis toward a primary malignant tumor of the pericardium and characterize its heterogeneity.

\section{DISCLOSURE}

No potential conflict of interest relevant to this article was reported.

\section{REFERENCES}

1. Benz MR, Czernin J, Dry SM, et al. Quantitative F18-fluorodeoxyglucose positron emission tomography accurately characterizes peripheral nerve sheath tumors as malignant or benign. Cancer. 2010;116:451-458.

2. Khiewvan B, Macapinlac HA, Lev D, et al. The value of ${ }^{18} \mathrm{~F}$-FDG PET/CT in the management of malignant peripheral nerve sheath tumors. Eur J Nucl Med Mol Imaging. 2014;41:1756-1766.

3. Maurer AH, Burshteyn M, Adler LP, Steiner RM. How to differentiate benign versus malignant cardiac and paracardiac ${ }^{18} \mathrm{~F}$ FDG uptake at oncologic PET/CT. Radiographics. 2011;31:1287-1305.

4. Warbey VS, Ferner RE, Dunn JT, Calonje E, O'Doherty MJ. $\left[{ }^{18}\right.$ F $]$ FDG PET/CT in the diagnosis of malignant peripheral nerve sheath tumours in neurofibromatosis type-1. Eur J Nucl Med Mol Imaging. 2009;36:751-757.

5. Salamon J, Veldhoen S, Apostolova I, et al. ${ }^{18}$ F-FDG PET/CT for detection of malignant peripheral nerve sheath tumours in neurofibromatosis type 1: tumourto-liver ratio is superior to an SUVmax cut-off. Eur Radiol. 2014;24:405-412. 